\title{
Estudo colaborativo para avaliação dos teores de proteína bruta em alimentos utilizando o método de Kjeldhal
}

\author{
Collaborative study to evaluate of the crude protein content in feeds using the \\ "Kjeldahl" method
}

\author{
SOUZA, Marjorrie Augusto de ${ }^{1}$; DETMANN, Edenio ${ }^{1 *}$; FRANCO, Marcia de \\ Oliveira $^{1}$; BATISTA, Erick Darlisson ${ }^{1}$; ROCHA, Gabriel Cipriano ${ }^{1}$; VALADARES \\ FILHO, Sebastião de Campos ${ }^{1}$; SALIBA, Eloísa de Oliveira Simões ${ }^{2}$
}

\footnotetext{
${ }^{1}$ Universidade Federal de Viçosa, Departamento de Zootecnia, Viçosa, Minas Gerais, Brasil.

${ }^{2}$ Universidade Federal de Minas Gerais, Escola de Veterinária, Belo Horizonte, Minas Gerais, Brasil.

*Endereço para correspondência: detmann@ufv.br
}

\section{RESUMO}

Estudo colaborativo foi conduzido para avaliação do teor de proteína bruta (PB) em diferentes amostras de alimentos em sete diferentes laboratórios de análise de alimentos de instituições integrantes do Instituto Nacional de Ciência e Tecnologia de Ciência Animal (INCT-CA). Foram utilizadas amostras de quatro alimentos (cana-de-açúcar, silagem de milho, farelo de soja e milho grão) e quatro compostos que foram assumidos como padrões para aferição da exatidão das estimativas (ureia, fosfato de amônia monobásico, ácido etileno diamínico tetra-acético e sulfato de amônio). Cada laboratório analisou as amostras durante seis dias, consecutivos ou não, sendo realizadas três repetições por amostra em cada dia de avaliação. As variâncias entre laboratórios e dentro de laboratórios (repetibilidade) representaram 34,5 e $10,3 \%$ da variação total. A avaliação das recuperações de nitrogênio a partir dos padrões permitiu evidenciar que $25 \%$ das observações apresentaram vícios significativos de recuperação $(\mathrm{P}<0,001)$. A avaliação global das amostras propiciou variações significativas $(\mathrm{P}<0,001)$ em função: do dia de análise, interação do material com o laboratório e entre laboratórios. Adicionalmente, verificou-se que a variação dos resultados entre laboratórios correspondeu de 43,6 a $68,2 \%$ da variação aleatória total, ao passo que a repetibilidade (variação intralaboratorial) representou de 11,9 a $42,8 \%$ da reprodutibilidade. Os teores de $\mathrm{PB}$ estimados pelos laboratórios participantes são dependentes do efeito de interação do material avaliado e do laboratório, possível reflexo das várias diferenças entre os procedimentos utilizados por cada laboratório.

Palavras-chave: análise de alimentos, laboratório, nitrogênio, repetibilidade, reprodutibilidade

\section{SUMMARY}

A collaborative study was conducted to evaluate the crude protein (CP) content in feeds in seven feed analysis laboratories from institutions linked to the National Institute of Science and Technology in Animal Science (INCT-CA). Were evaluated four samples of different feeds (sugarcane, corn silage, soybean meal and corn grain) and four nitrogen compounds assumed as standards (urea, monobasic ammonium phosphate, ethylene diamine tetraacetic acid and ammonium sulfate) to measure the accuracy of the estimates. Each laboratory analyzed the samples for six days, consecutive or not, with three replicates per sample per day of evaluation. The variances among laboratories and within of laboratories (repeatability) represented 34.5 and $10.3 \%$ of the total variation, respectively. The evaluation of nitrogen recovered from the standards showed that $25 \%$ of observations presented a significant bias $(\mathrm{P}<0.001)$. The overall assessment of the samples demonstrated significant variations $(\mathrm{P}<0.001)$ in function of the day of analysis, 
interaction of material with laboratory, and among laboratories. Additionally, it was found that the variation among laboratories corresponded of 43.6 to $68.2 \%$ of the total of random variation, while the repeatability (intralaboratory variation) represented of 11.9 to $42.8 \%$ of reproducibility. The CP contents estimated are dependent of the interaction effect of the evaluated material and laboratory, which reflect the differences between the procedures utilized by each laboratory.

Keywords: feed analysis, laboratory, nitrogen, repetibility, reprodutibility

\section{INTRODUÇÃO}

O termo proteína bruta (PB) envolve grande grupo de substâncias com estruturas semelhantes, porém com funções químicas e fisiológicas diferentes. Baseado no fato de as proteínas terem percentual de nitrogênio (N) aproximadamente constante (em torno de $16 \%$ ), procede-se à sua avaliação indireta por intermédio da concentração de $\mathrm{N}$ no material e utilizando-se o fator de conversão 6,25 para a conversão do resultado em equivalente proteico (SILVA \& QUEIROZ, 2002).

O método mais adotado no Brasil foi proposto por Kjeldahl na Dinamarca em 1883, quando estudava proteína em grãos. Este apresenta três etapas distintas: digestão, destilação e titulação. A digestão baseia-se no aquecimento da amostra com ácido sulfúrico até que os compostos orgânicos sejam oxidados. $\mathrm{O}$ $\mathrm{N}$ da proteína (orgânico) é reduzido e transformado em sulfato de amônia (inorgânico) que é uma substância estável (POMERANZ \& MELOAN, 1978), porém não facilmente quantificável. Nessa etapa utiliza-se uma mistura digestora, composta de um sal (sulfato de sódio ou de potássio) com a finalidade de elevar o ponto de ebulição do ácido sulfúrico permitido a decomposição da matéria orgânica, e um catalizador metálico que aumenta o poder de oxidação do meio. Posteriormente, adiciona-se hidróxido de sódio concentrado e aquece-se para a liberação da amônia em solução de ácido bórico, formando borato ácido de amônia que constitui forma quantificável do N. O borato de amônia formado é titulado com uma solução padronizada de ácido clorídrico.

Em muitos laboratórios estão sendo implementados fundamentos de controle de qualidade para se ampliar a exatidão e a precisão dos resultados produzidos. Para auxiliar nesta meta torna-se importante que os métodos oficialmente aprovados sejam adotados para que a tecnologia avaliada seja empregada nos laboratórios de modo que os resultados produzidos possam ser comparados (THIEX \& MANSON, 2002).

Desta forma, dada a importância da PB obtida a partir do método de Kjeldhal, faz-se necessária a avaliação dos procedimentos laboratoriais e das estimativas de PB produzidas em diferentes materiais e por diferentes laboratórios, buscando-se identificar variantes mais adequadas e padronizar os procedimentos entre laboratórios.

Objetivou-se conduzir estudo colaborativo para avaliação da repetibilidade e reprodutibilidade na determinação do teor de PB de diferentes materiais em sete laboratórios de análise de alimentos de instituições integrantes do Instituto Nacional de Ciência e Tecnologia de Ciência Animal (INCTCA).

\section{MATERIAL E MÉTODOS}

As avaliações foram conduzidas em sete laboratórios de análise de alimentos de instituições componentes do INCT-CA: Universidade Federal de Viçosa, Viçosa- 
Rev. Bras. Saúde Prod. Anim., Salvador, v.17, n.4, p.696-709 out./dez., 2016 http://www.rbspa.ufba.br ISSN 15199940

MG; Escola de Veterinária da Universidade Federal de Minas Gerais, Belo Horizonte-MG; Universidade Federal de Lavras, Lavras-MG; Universidade Estadual de Santa Cruz, Ilhéus-BA; Universidade Estadual Paulista Júlio de Mesquita, JaboticabalSP; Universidade Federal de Mato Grosso, Cuiabá-MT; e Universidade Federal de Mato Grosso, Sinop-MT; entre janeiro e março de 2010.

Foram utilizadas amostras de quatro alimentos: cana-de-açúcar, silagem de milho, farelo de soja e milho grão. Em adição, foram enviados quatro compostos, assumidos como padrões para aferição da exatidão das estimativas: ureia (Sigma U5378), fosfato de amônia monobásico (MAP; Sigma-Aldrich A1645), ácido etileno diamínico tetra-acético (EDTA; Sigma-Aldrich EDS) e sulfato de amônio (Merk 01217).

As amostras de volumosos úmidos foram secas em estufa com ventilação forçada $\left(60^{\circ} \mathrm{C}\right)$ e, em conjunto com as demais amostras, processadas em moinho de facas $(1 \mathrm{~mm})$, de acordo com Silva e Queiroz (2002). Em seguida as amostras foram acondicionadas em sacos plásticos e enviadas sem identificação, juntamente com os padrões, aos laboratórios. $\mathrm{Na}$ oportunidade do envio solicitou-se a cada laboratório que as amostras fossem analisadas por seis dias, consecutivos ou não, sendo realizadas três repetições por amostra por dia de avaliação.

Os resultados foram solicitados com base na amostra seca ao ar, uma vez que a avaliação de resultados com correção para o teor de matéria seca de cada amostra entre laboratórios pode apresentar erro oriundo de dois procedimentos (MERTENS, 2003). Em conjunto com as amostras, enviou-se questionário para elucidação dos principais procedimentos e parâmetros analíticos quantitativos e qualitativos adotados em cada laboratório. Os dados foram analisados considerandose padrões (em termos de recuperação do N) e amostras (em termos de teor estimado de PB) separadamente, de acordo com o modelo:

$Y_{i j k l}=\mu+L_{i}+M_{j}+L M_{i j}+D_{(i j) k}+\varepsilon_{(i j k) l}$

em que: $\mathrm{Y}_{\mathrm{ijkl}}=$ teor de PB (\%) obtida no laboratório $\mathrm{i}$, no material $\mathrm{j}$, na repetição $\mathrm{k}$ avaliada no dia de análise $\mathrm{j} ; \mu=$ constante geral; $\mathrm{L}_{\mathrm{i}}=$ efeito do laboratório $\mathrm{i}$ (aleatório); $\mathrm{M}_{\mathrm{j}}=$ efeito do material $\mathrm{j}$ (fixo); $\mathrm{LM}_{\mathrm{ij}}=$ efeito de interação do laboratório i e material j (aleatório); $\mathrm{D}_{(\mathrm{ij}) \mathrm{k}}$ $=$ efeito do dia de análise $\mathrm{k}$, aninhado à combinação entre o laboratório i e o material $\mathrm{j}$ (aleatório); e $\varepsilon_{(\mathrm{ijk}) 1}=$ erro aleatório, associado a cada mensuração, pressuposto NID $\left(0 ; \sigma_{\varepsilon}^{2}\right)$.

Por intermédio do método dos momentos, a partir dos fundamentos do procedimento de Hicks modificado (BARBIN, 1993), definiram-se as esperanças de quadrados médios da análise de variância dos padrões e amostras, conforme apresentado na Tabela 1.

As observações classificadas como outliers foram eliminadas do conjunto de dados, sendo estas definidas quando seu desvio em relação à média das avaliações do material, dentro do laboratório, superasse em três vezes o valor do desvio padrão residual.

A partir das esperanças de quadrados médios (Tabela 1) estimaram-se a repetibilidade $(r)$ e a reprodutibilidade (R) dos teores de PB, conforme descrito por Mertens (2003):

$r=\hat{\sigma}_{\varepsilon}^{2}$
$R=\hat{\sigma}_{\varepsilon}^{2}+\hat{\sigma}_{L}^{2}$ 
Rev. Bras. Saúde Prod. Anim., Salvador, v.17, n.4, p.696-709 out./dez., 2016 http://www.rbspa.ufba.br ISSN 15199940

Tabela 1. Esperança de quadrados médios para modelo destinado à análise global dos dados

\begin{tabular}{|c|c|}
\hline Fonte de Variação $^{1}$ & $\mathrm{E}(\mathrm{QM})^{2}$ \\
\hline $\mathrm{L}$ & $\sigma_{\varepsilon}^{2}+\mathrm{k}_{1} \times \sigma_{\mathrm{D} / \mathrm{L} \times \mathrm{M}}^{2}+\mathrm{k}_{2} \times \sigma_{\mathrm{L} \times \mathrm{M}}^{2}+\mathrm{k}_{4} \times \sigma_{\mathrm{L}}^{2}$ \\
\hline $\mathrm{M}$ & $\sigma_{\varepsilon}^{2}+k_{1} \times \sigma_{D / L \times M}^{2}+k_{2} \times \sigma_{L \times M}^{2}+k_{3} \times \phi_{M}$ \\
\hline $\mathrm{L} \times \mathrm{M}$ & $\sigma_{\varepsilon}^{2}+k_{1} \times \sigma_{D / L \times M}^{2}+k_{2} \times \sigma_{L \times M}^{2}$ \\
\hline $\mathrm{D} / \mathrm{L} \times \mathrm{M}$ & $\sigma_{\varepsilon}^{2}+\mathrm{k}_{1} \times \sigma_{\mathrm{D} / \mathrm{L} \times \mathrm{M}}^{2}$ \\
\hline Resíduo & $\sigma_{\varepsilon}^{2}$ \\
\hline \multicolumn{2}{|c|}{$\begin{array}{l}{ }^{1} \mathrm{~L}=\text { laboratório; } \mathrm{M}=\text { material; } \mathrm{L} \times \mathrm{M}=\text { interação laboratório } \times \text { material, } \mathrm{D} / \mathrm{L} \times \mathrm{M}=\text { dia aninhado às } \\
\text { combinações entre laboratório e material; }{ }^{2} \sigma_{\varepsilon}^{2}, \sigma^{2} \mathrm{D} / \mathrm{L} \times \mathrm{M}, \sigma_{\mathrm{L} \times \mathrm{M}}, \sigma^{2} \mathrm{~L}=\text { variâncias associadas aos efeitos do } \\
\text { erro (repetibilidade), de dia aninhado às combinações entre laboratório e material, da interação de } \\
\text { laboratório e material e de laboratório, respectivamente; } \phi_{\mathrm{M}}=\text { função quadrática associada ao efeito fixo } \\
\text { de material. }\end{array}$} \\
\hline
\end{tabular}

Quando o efeito de interação de material analisado e laboratório foi significativo, procedeu-se a nova avaliação, na qual considerou-se cada material isoladamente. Esclarece-se que na presença de interação, tanto a reprodutibilidade como a repetibilidade estimadas a partir dos componentes de variância obtidos na equação (1) não podem ser utilizadas para representar o comportamento de cada material em geral. Como são avaliados efeitos aleatórios não é possível realizar o desdobramento em um único modelo. Cabe ressaltar, que efeitos aleatórios não constituem parâmetros da população, mas sim constituem variáveis aleatórias contínuas. Desta forma, um componente de variância comum para laboratório não poderá ser usado para a avaliação de cada um dos materiais. Como o desdobramento em um único modelo é impossível neste caso, a estimação de componentes de variâncias individuais só é possível com o fracionamento do banco de dados. Desta forma, o desdobramento foi realizado utilizando-se um modelo para avaliação da variação de cada material considerando-se os diferentes laboratórios:

$$
Y_{i j k}=\mu+L_{i}+D_{(i) j}+\varepsilon_{(i j) k}
$$

em que: $\mathrm{Y}_{\mathrm{ijk}}=$ teor de PB (\%) obtida no laboratório $\mathrm{i}$, na repetição $\mathrm{k}$ avaliada no dia de análise $\mathrm{j} ; \mu=$ constante geral; $\mathrm{L}_{\mathrm{i}}$ $=$ efeito do laboratório i (aleatório); $\mathrm{D}_{(\mathrm{i}) \mathrm{j}}$ $=$ efeito do dia de análise $\mathrm{j}$ aninhado ao laboratório i (aleatório); e $\varepsilon_{(\mathrm{ij}) \mathrm{k}}=$ erro aleatório, associado a cada mensuração, pressuposto NID $\left(0 ; \sigma_{\varepsilon}^{2}\right)$.

As esperanças de quadrados médios para o modelo descrito em (4) são descritas na Tabela 2. As estimativas de repetibilidade e reprodutibilidade para cada material foram obtidas de forma similar ao descrito nas Equações (2) e (3).

Para a situação descrita na Tabela 2, procedeu-se à avaliação das estimativas de reprodutibilidade esperada e da razão de Horwitz (HORWITZ et al., 1990):

$$
\begin{aligned}
& R p=\frac{\hat{\sigma}_{R}}{\bar{X}} \\
& \hat{\sigma}_{R}=\sqrt{R} \\
& R p e=2 \exp (1-0,5 \log c) \\
& R H=\frac{R p}{R p e}
\end{aligned}
$$

em que: $\mathrm{Rp}=$ reprodutibilidade padronizada em função da média; Rpe = reprodutibilidade padronizada em função da média estimada pelo método de Horwitz; c = concentração média de PB $(\mathrm{g} / \mathrm{g})$; e RH = razão de Horwitz. 
Rev. Bras. Saúde Prod. Anim., Salvador, v.17, n.4, p.696-709 out./dez., 2016 http://www.rbspa.ufba.br ISSN 15199940

Tabela 2. Esperanças de quadrados médios para o modelo destinado à análise de cada material em função dos diferentes laboratórios (Equação 4)

\begin{tabular}{ll}
\hline $\begin{array}{l}\text { Fonte de } \\
\text { Variação }\end{array}$ & \multicolumn{1}{c}{$\mathrm{E}(\mathrm{QM})^{2}$} \\
\hline $\mathrm{L}$ & $\sigma_{\varepsilon}^{2}+\mathrm{k}_{1} \times \sigma^{2} \mathrm{D} / \mathrm{L}+\mathrm{k}_{2} \times \sigma_{\mathrm{L}}^{2}$ \\
$\mathrm{D} / \mathrm{L}$ & $\sigma_{\varepsilon}^{2}+\mathrm{k}_{1} \times \sigma_{\mathrm{D} / \mathrm{L}}$ \\
Resíduo & $\sigma_{\varepsilon}^{2}$ \\
\hline${ }^{1} \mathrm{~L}=$ laboratório; $\mathrm{D} / \mathrm{L}=$ dia aninhado ao \\
laboratório; ${ }^{2} \sigma_{\varepsilon}^{2}, \sigma^{2} \mathrm{D} / \mathrm{L}, \sigma^{2} \mathrm{~L}=$ variâncias \\
associadas aos efeitos do erro (repetibilidade), \\
de dia aninhado ao laboratório e de \\
laboratório, respectivamente.
\end{tabular}

Quando o efeito de dia de análise foi significativo, procedeu-se ao desdobramento das avaliações conforme explicações apresentadas anteriormente, com a avaliação do comportamento de cada material em cada laboratório por intermédio do modelo:

$Y_{i j}=\mu+D_{i}+\varepsilon_{(i) j}$

em que: $\mathrm{Y}_{\mathrm{ij}}=$ teor de PB (\%) obtida na repetição $\mathrm{j}$ avaliada no dia de análise $\mathrm{i} ; \mu$ $=$ constante geral; $\mathrm{D}_{\mathrm{i}}=$ efeito do dia de análise i (aleatório); e $\varepsilon_{(\mathrm{i}) \mathrm{j}}=$ erro aleatório, associado a cada mensuração, pressuposto NID $\left(0 ; \sigma_{\varepsilon}^{2}\right)$.

Por intermédio do método dos momentos (BARBIN, 1993), definiram-se as esperanças de quadrados médios associadas ao modelo descrito em (9) (Tabela 3).

A variação aleatória total associada ao modelo descrito em (9) foi dada por:

$\hat{\sigma}_{T}^{2}=\hat{\sigma}_{D}^{2}+\hat{\sigma}_{\varepsilon}^{2}$

Todos os procedimentos estatísticos foram conduzidos por intermédio do programa SAS versão 9.2 (Statisical Analysis System), adotando-se 0,001 como nível crítico de probabilidade para o erro tipo I. As comparações entre médias fornecidas por diferentes laboratórios foram conduzidas utilizando-se o critério de Scheffé. Por outro lado, a avaliação da recuperação de $\mathrm{N}$ nos diferentes padrões de acordo com os laboratórios foi realizada utilizando-se as propriedades de intervalos de confiança $(1-\alpha=0,999)$.

Tabela 3. Esperanças de quadrados médios para o modelo destinado à análise de cada material em cada laboratório em função dias de análise (Equação 9)

\begin{tabular}{lc}
\hline Fonte de Variação $^{1}$ & $\mathrm{E}(\mathrm{QM})^{2}$ \\
\hline $\mathrm{D}$ & $\sigma^{2}{ }_{\varepsilon}+\mathrm{k}_{1} \times \sigma^{2} \mathrm{D}$ \\
Resíduo & $\sigma^{2}{ }_{\varepsilon}$ \\
\hline${ }^{1} \mathrm{D}=$ dia; ${ }^{2} \sigma^{2}{ }_{\varepsilon}, \sigma^{2}{ }_{\mathrm{D}}=$ variâncias associadas aos \\
efeitos do erro e de dia de análise, \\
respectivamente.
\end{tabular}

\section{RESULTADOS E DISCUSSÃO}

As variâncias entre laboratórios e intralaboratório (repetibilidade), considerando os padrões, representaram aproximadamente 34,5 e $10,3 \%$ da variação total (Tabela 4) e todos os componentes de variância foram significativos $(\mathrm{P}<0,001)$. Isto indica dois entraves nos resultados: a relação entre recuperação e dia de análise e a ocorrência de interação do material analisado com o laboratório no qual a análise é realizada.

A partir da avaliação das recuperações de $\mathrm{N}$ a partir dos padrões, evidenciou-se que $25 \%$ das observações apresentaram vícios negativos significativos de recuperação $(\mathrm{P}<0,001)$, indicando perda de $\mathrm{N}$ durante o processo analítico (Tabela 5). 
Rev. Bras. Saúde Prod. Anim., Salvador, v.17, n.4, p.696-709 out./dez., 2016 http://www.rbspa.ufba.br ISSN 15199940

Tabela 4. Estimativas de componentes de variância do modelo global (padrões)

\begin{tabular}{lccc}
\hline Item $^{1}$ & Estimativa & Valor relativo (\%) & Valor-P \\
\hline$\sigma^{2}$ & 0,0010 & 34,5 & 0,0004 \\
$\sigma^{2}{ }_{\text {LxM }}$ & 0,0005 & 17,3 & $<0,0001$ \\
$\sigma_{\text {D/LxM }}^{2}$ & 0,0011 & 37,9 & $<0,0001$ \\
Repetibilidade $\left(\sigma_{\varepsilon}^{2}\right)$ & 0,0003 & 10,3 & - \\
Variância Total & 0,0029 & 100,0 & - \\
Reprodutibilidade & 0,0013 & - & - \\
r/R & 0,2308 & - & - \\
\hline
\end{tabular}

${ }^{1} \sigma_{\mathrm{L}}^{2}, \sigma_{\mathrm{L} \times \mathrm{M}}^{2}$ e $\sigma_{\mathrm{D} / \mathrm{L} \times \mathrm{M}}^{2}=$ variâncias associadas aos efeitos do laboratório, da interação de laboratório e material e de dia aninhado às combinações entre laboratório e material, respectivamente; $r / R=$ Razão repetibilidade/reprodutibilidade.

Tabela 5. Recuperação de nitrogênio $(\mathrm{g} / \mathrm{g})$ a partir da composição química teórica de ureia, fosfato de amônia monobásico (MAP), ácido etileno diamínico tetraacético (EDTA) e sulfato de amônio pelos diferentes laboratórios

\begin{tabular}{lcccc}
\hline \multirow{2}{*}{ Item } & \multicolumn{3}{c}{ Padrão $^{4}$} \\
\cline { 2 - 5 } & Ureia & MAP & EDTA & Sulfato de Amônio. \\
\hline Teórico $^{1}$ & 291,67 & 76,09 & 59,93 & 132,58 \\
MS $^{2}$ & 99,76 & 99,74 & 99,74 & 99,69 \\
Esperado $^{3}$ & 290,96 & 75,89 & 59,78 & 132,17 \\
Laboratório 1 $^{\text {Laboratório 2 }}$ & 0,9840 & 0,9830 & 0,9606 & 0,9554 \\
Laboratório 3 & 1,0041 & 1,0030 & $0,8961^{*}$ & 1,0065 \\
Laboratório 4 & 0,9565 & 0,9846 & 0,9807 & 0,9758 \\
Laboratório 5 & $0,9008^{*}$ & $0,9340^{*}$ & $0,9420^{*}$ & $0,9217^{*}$ \\
Laboratório 6 & 0,9868 & 1,0264 & 1,0103 & 0,9963 \\
Laboratório 7 & 1,0424 & 1,0298 & 1,0240 & 1,0461 \\
\hline Percentual & $0,9460^{*}$ & 0,9915 & 0,9670 & $0,9313^{*}$ \\
\hline
\end{tabular}

${ }^{1}$ Percentual estimado a partir do peso atômico e composição molecular.

${ }^{2}$ Percentual de matéria seca avaliado pelo método de Karl Fischer (BRUTTEL \& SCHLINK, 2006).

${ }^{3}$ Percentual dado pelo produto entre a composição teórica e o teor de matéria seca.

${ }^{4}$ Médias seguidas por (*) apresentam vício de recuperação significativo em relação ao teor esperado do padrão utilizando-se as propriedades do intervalo de confiança $[(1-\alpha)=0,999]$.

A avaliação global das amostras propiciou comportamento similar ao observado para os padrões, com variações significativas $(\mathrm{P}<0,001) \mathrm{em}$ função do dia de análise e a presença de interação $(\mathrm{P}<0,001)$ do material com $\mathrm{o}$ laboratório. Assim, tanto os alimentos como os padrões foram analisados diferentemente em cada laboratório e as estimativas dos teores de N/PB se mostram dependentes do dia em que a análise foi realizada (Tabela 6).

De forma geral, assume-se como não aceitável a ocorrência de efeito de interação de material e laboratório (MERTENS, 2003). A ocorrência deste indica que os possíveis erros sistemáticos imputados a um dado material em determinado laboratório serão distintos daqueles observados em outro laboratório. 
Rev. Bras. Saúde Prod. Anim., Salvador, v.17, n.4, p.696-709 out./dez., 2016 http://www.rbspa.ufba.br ISSN 15199940

Tabela 6. Estimativas de componentes de variância do modelo global (amostras)

\begin{tabular}{lccc}
\hline Item $^{1}$ & Estimativa & Valor relativo (\%) & Valor-P \\
\hline$\sigma_{\mathrm{L}}^{2}$ & 0,0683 & 6,2 & 0,2446 \\
$\sigma_{\mathrm{LxM}}^{2}$ & 0,5288 & 48,3 & $<0,0001$ \\
$\sigma_{\mathrm{D} / \mathrm{LxM}}$ & 0,2472 & 22,6 & $<0,0001$ \\
Repetibilidade $\left(\sigma_{\varepsilon}^{2}\right)$ & 0,2514 & 22,9 & - \\
Variância Total & 1,0957 & 100,0 & - \\
Reprodutibilidade & 0,3197 & - & - \\
$\mathrm{r} / \mathrm{R}$ & 0,7864 & - & - \\
Média & 15,70 & - & - \\
\hline${ }^{1} \sigma_{\mathrm{L}}^{2}, \sigma_{\mathrm{L} \times \mathrm{M}}^{2}$ e $\sigma^{2} \mathrm{D} / \mathrm{L} \times \mathrm{M}=$ variâncias associadas aos efeitos do laboratório, da interação de laboratório e \\
material e de dia aninhado às combinações entre laboratório e material, respectivamente; r/R = Razão \\
repetibilidade/reprodutibilidade.
\end{tabular}

A isto se adiciona o fato de que os erros sistemáticos imputados por um laboratório se manifestarão de forma diferenciada em função do material analisado. Isto atribui elemento de confundimento aos cotejamentos dos conteúdos de PB de alimentos e outros materiais, como fezes, sobras e digestas; o que, consequentemente, pode comprometer a comparação entre características obtidas em ensaios de consumo, digestão, degradação e metabolismo.
A interação de material e laboratório é corroborada pela avaliação comparativa das médias obtidas nos diferentes alimentos, na qual se observou variação entre laboratórios, não havendo padrão definido nos perfis de comparações múltiplas. Verificou-se que as diferenças entre laboratórios variaram de 0,50 ponto percentual para o milho a 4,25 pontos percentuais para o farelo de soja (Tabela 7).

Tabela 7. Comparação entre médias produzidas para cana-de-açúcar, silagem de milho (SM), farelo de soja (FS) e milho grão pelos diferentes laboratórios

\begin{tabular}{lcccc}
\hline Laboratório $^{2}$ & \multicolumn{4}{c}{ Material $^{1}$} \\
\cline { 2 - 5 } & Cana & SM & FS & Milho \\
\hline 1 & $3,67^{\mathrm{ab}}$ & $6,75^{\mathrm{abc}}$ & $43,68^{\mathrm{ab}}$ & $7,55^{\mathrm{ab}}$ \\
2 & $3,61^{\mathrm{ab}}$ & $7,11^{\mathrm{ab}}$ & $44,77^{\mathrm{ab}}$ & $7,43^{\mathrm{ab}}$ \\
3 & $4,02^{\mathrm{a}}$ & $7,06^{\mathrm{ab}}$ & $43,45^{\mathrm{ab}}$ & $7,71^{\mathrm{ab}}$ \\
4 & $3,49^{\mathrm{b}}$ & $6,14^{\mathrm{c}}$ & $42,62^{\mathrm{b}}$ & $7,31^{\mathrm{b}}$ \\
5 & $3,71^{\mathrm{ab}}$ & $7,53^{\mathrm{a}}$ & $45,72^{\mathrm{ab}}$ & $7,81^{\mathrm{a}}$ \\
6 & $3,42^{\mathrm{b}}$ & $6,59^{\mathrm{bc}}$ & $46,87^{\mathrm{a}}$ & $7,69^{\mathrm{ab}}$ \\
7 & $3,63^{\mathrm{ab}}$ & $6,73^{\mathrm{abc}}$ & $45,87^{\mathrm{ab}}$ & $7,75^{\mathrm{ab}}$ \\
EPM $^{2}$ & 0,061 & 0,108 & 0,449 & 0,063 \\
$\mathrm{ATM}^{2}$ & 0,60 & 1,39 & 4,25 & 0,50 \\
\hline
\end{tabular}

${ }^{1}$ Médias na coluna, seguidas por letras diferentes, são diferentes pelo teste de Scheffé $(\mathrm{P}<0,001)$.

${ }^{2} \mathrm{EPM}$, erro padrão da média; ATM, amplitude total entre médias (diferença entre a maior e menor média de laboratórios). 
A avaliação das amostras individualmente evidenciou variação inter-laboratorial elevada para o farelo de soja em comparação às demais amostras (Figura 1). Considerando-se que este material possui o maior percentual de PB em comparação aos demais, os vícios de recuperação aqui observados causariam desvios mais proeminentes para o farelo de soja, o que parece justificar sua baixa reprodutibilidade.

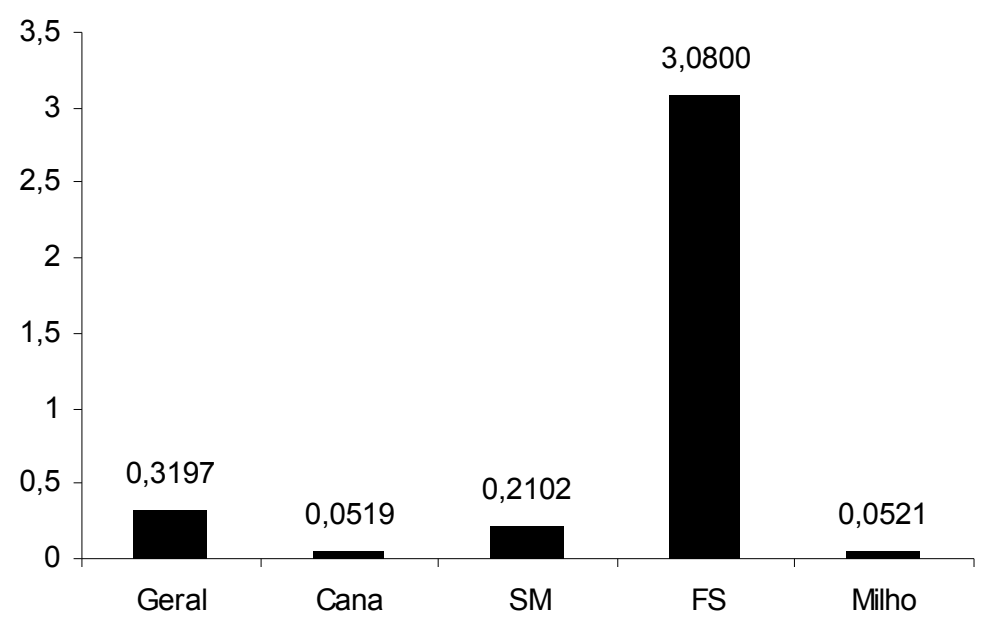

Figura 1. Reprodutibilidade $\left[(\%)^{2}\right]$ para os diferentes materiais analisados

Observou-se variação significativa entre laboratórios $(\mathrm{P}<0,001)$ para todos as amostras avaliadas (Tabela 8). Adicionalmente, verificou-se que a variação dos resultados entre laboratórios correspondeu de 43,6 a $68,2 \%$ da variação aleatória total, ao passo que a repetibilidade (variação intra-laboratorial) representou de 11,89 a $42,80 \%$ da reprodutibilidade (Tabela 8). O comportamento destes percentuais reitera que diferenças entre laboratórios constituem o fator preponderante das diferenças entre os teores de PB.

A avaliação da reprodutibilidade, a qual é dada pela soma da repetibilidade e da variação entre laboratórios apresentouse em patamares aceitáveis, pois se observou razões de Horwitz (RH) inferiores a 2 para todos os materiais avaliados (Tabela 8).

A partir dos dados expressos na Tabela 8 , as repetibilidades padronizadas (expressas como percentuais das médias) foram de 3,$84 ; 2,31 ; 2,17$; e 1,96\% para cana-de-açúcar, silagem de milho, capim-elefante, farelo de soja e milho, respectivamente. Estas estimativas indicam os baixos valores relativos da variação entre repetições. Logo, os baixos valores de RH (Tabela 8) parecem ter sido causados pela baixa variação entre repetições. No entanto, embora aparentemente em patamares adequados, os valores de RH podem não indicar completa adequação das estimativas de reprodutibilidade. 
Rev. Bras. Saúde Prod. Anim., Salvador, v.17, n.4, p.696-709 out./dez., 2016 http://www.rbspa.ufba.br ISSN 15199940

Tabela 8. Estimativas de componentes de variância do modelo destinado à avaliação individual dos materiais (Equação 4)

\begin{tabular}{|c|c|c|c|}
\hline Item & Estimativa & Valor relativo (\%) & Valor-P \\
\hline \multicolumn{4}{|c|}{ Cana-de-açúcar } \\
\hline$\sigma_{L}^{2}$ & 0,0322 & 47,4 & $<0,0001$ \\
\hline$\sigma_{\mathrm{D} / \mathrm{L}}^{2}$ & 0,0160 & 23,6 & $<0,0001$ \\
\hline Repetibilidade $\left(\sigma_{\varepsilon}^{2}\right)$ & 0,0197 & 29,0 & - \\
\hline Variância Total & 0,0679 & 100,0 & - \\
\hline Reprodutibilidade & 0,0519 & - & - \\
\hline $\mathrm{r} / \mathrm{R}$ & 0,3796 & - & - \\
\hline RH & 1,5200 & - & - \\
\hline Média & 3,6521 & - & - \\
\hline \multicolumn{4}{|c|}{ Silagem de milho } \\
\hline$\sigma_{\mathrm{L}}^{2}$ & 0,1852 & 68,2 & $<0,0001$ \\
\hline$\sigma_{\mathrm{D} / \mathrm{L}}^{2}$ & 0,0614 & 22,6 & $<0,0001$ \\
\hline Repetibilidade $\left(\sigma_{\varepsilon}^{2}\right)$ & 0,0250 & 9,2 & - \\
\hline Variância Total & 0,2716 & 100 & - \\
\hline Reprodutibilidade & 0,2102 & - & - \\
\hline $\mathrm{r} / \mathrm{R}$ & 0,1189 & - & - \\
\hline RH & 1,8712 & - & - \\
\hline Média & 6,8435 & - & - \\
\hline \multicolumn{4}{|c|}{ Farelo de soja } \\
\hline$\sigma_{\mathrm{L}}^{2}$ & 2,1413 & 53,9 & $<0,0001$ \\
\hline$\sigma_{\mathrm{D} / \mathrm{L}}^{2}$ & 0,8952 & 22,5 & $<0,0001$ \\
\hline Repetibilidade $\left(\sigma_{\varepsilon}^{2}\right)$ & 0,9387 & 23,6 & - \\
\hline Variância Total & 3,9752 & 100,0 & - \\
\hline Reprodutibilidade & 3,0800 & - & - \\
\hline $\mathrm{r} / \mathrm{R}$ & 0,3048 & - & - \\
\hline RH & 1,6478 & - & - \\
\hline Média & 44,7127 & - & - \\
\hline \multicolumn{4}{|c|}{ Milho grão } \\
\hline$\sigma_{L}^{2}$ & 0,0298 & 43,6 & $<0,0001$ \\
\hline$\sigma_{\mathrm{D} / \mathrm{L}}^{2}$ & 0,0163 & 23,8 & $<0,0001$ \\
\hline Repetibilidade $\left(\sigma_{\varepsilon}^{2}\right)$ & 0,0223 & 32,6 & - \\
\hline Variância Total & 0,0684 & 100,0 & - \\
\hline Reprodutibilidade & 0,0521 & - & - \\
\hline $\mathrm{r} / \mathrm{R}$ & 0,4280 & - & - \\
\hline RH & 0,8577 & - & - \\
\hline Média & 7,6063 & - & - \\
\hline
\end{tabular}

Considerando-se as informações constantes na Tabela 8 , percebeu-se que para todos os materiais ocorreu efeito significativo do dia de análise $(\mathrm{P}<0,001)$ sobre as estimativas de PB. Contudo, a avaliação isolada deste efeito para cada material analisado em cada laboratório indicou que apenas quatro das 28 avaliações apresentaram variações significativas $(\mathrm{P}<0,001)$ das estimativas de $\mathrm{PB}$ em função do dia no qual a análise foi realizada (Tabela 9), fração que deve ser considerada pequena em 
Rev. Bras. Saúde Prod. Anim., Salvador, v.17, n.4, p.696-709 out./dez., 2016 http://www.rbspa.ufba.br

comparação a outras avaliações conduzidas neste estudo colaborativo.

A variabilidade ao longo dos dias de análise implica que os resultados obtidos no processo analítico são diretamente dependentes deste fator, o que, obviamente, pode atribuir efeito de confundimento sobre a composição química do material analisado. Por lógica, evidencia-se que problema atribuído à falta de padronização dos procedimentos intra-laboratoriais somase a questão da alta variabilidade interlaboratorial sobre a confiabilidade dos resultados de PB.

Tabela 9. Nível descritivo de probabilidade para o erro tipo I associada à variação entre dias de análises para cana-de-açúcar, silagem de milho (SM), farelo de soja (FS) e milho grão

\begin{tabular}{ccccc}
\hline \multirow{2}{*}{ Laboratório } & \multicolumn{4}{c}{ Material $^{1}$} \\
\cline { 2 - 5 } & Cana & SM & FS & Milho \\
\hline 1 & 0,0869 & 0,1062 & 0,0030 & 0,0174 \\
2 & 0,9726 & 0,5488 & 0,5439 & 0,7194 \\
3 & 0,0227 & 0,0014 & $<0,0001$ & 0,0705 \\
4 & 0,0638 & 0,0046 & 0,9494 & 0,1635 \\
5 & 0,0041 & $<0,0001$ & 0,1689 & 0,0191 \\
6 & 0,9912 & 0,0059 & 0,0954 & 0,0004 \\
7 & 0,0298 & 0,2116 & $<0,0001$ & 0,3392 \\
\hline
\end{tabular}

Elevada variabilidade foi observada nos métodos adotados pelos diferentes laboratórios (Tabelas 10 e 11), o que evidencia possíveis causas da variação dos resultados (Tabela 8) e do efeito de interação de laboratório e material (Tabelas 4 e 6).

Em nenhuma das características do processo de digestão se verificou homogeneidade entre laboratórios (Tabela 10). As relações entre volume de ácido e amostra, entre mistura digestora e ácido e entre mistura digestora e amostra variaram de 2 a $5 \mathrm{~mL} / 100 \mathrm{mg}$, de 0,3 a $0,5 \mathrm{~g} / \mathrm{mL}$ e de 0,6 a $1,25 \mathrm{~g} / 100$ mg, respectivamente. Embora nenhum dos valores possa ser considerado extremo, a variabilidade das relações entre laboratórios pode ter contribuído para a variação observada neste estudo.

Todos os laboratórios utilizam sulfato de cobre como catalisador metálico, exceto o laboratório 5, no qual selenito de sódio é utilizado conjuntamente. $\mathrm{O}$ uso de selênio como catalisador metálico pode acelerar o processo de digestão; contudo este pode ocasionar perdas de $\mathrm{N}$ durante a digestão se for utilizado em excesso ou se a temperatura não for rigorosamente controlada, o que torna mais críticas as condições analíticas com a sua utilização (POMERANZ \& MELOAN, 1978; CECCHI, 2003).

Embora o cobre seja considerado catalisador de baixa eficiência comparado ao mercúrio ou selênio (CECCHI, 2003), sua utilização é recomendada principalmente pelo menor custo e por não requerer etapas analíticas adicionais (como o mercúrio) e não demandar controle rigoroso das condições de digestão (como o selênio).

Nos laboratórios utilizaram-se os sulfatos de sódio ou de potássio como sais para elevação da temperatura de ebulição do ácido sulfúrico. Embora o sulfato de potássio tenha sido inicialmente proposto como sal para essa finalidade 
Rev. Bras. Saúde Prod. Anim., Salvador, v.17, n.4, p.696-709 out./dez., 2016 http://www.rbspa.ufba.br ISSN 15199940

(modificação de Gunning de 1889; POMERANZ \& MELOAN, 1978), admite que o sulfato de sódio apresente efeito similar, sendo também recomendado (SILVA \& QUEIROZ, 2002).

A relação sal:catalisador na mistura digestora foi relativamente constante entre laboratórios, com exceção do laboratório 3, cuja relação foi extremamente alta. Isto indica baixa concentração do catalisador na mistura digestora, o que pode elevar demasiadamente o tempo de digestão.

As temperaturas de digestão variaram de 350 a $430^{\circ} \mathrm{C}$. Existem diversas recomendações de temperaturas de extração relatadas na literatura, algumas relevando particularidades do processo de digestão. Faithfull (2002) recomendou temperaturas de $310^{\circ} \mathrm{C}$, com tolerância de no máximo $320^{\circ} \mathrm{C}$. Contudo, este autor sugeriu somente o uso de selenito de sódio em conjunto com o ácido sulfúrico, cuja temperatura de ebulição passaria a ser de $330^{\circ} \mathrm{C}$.

Considerando-se o uso de sal (sulfato de sódio ou potássio), Silva \& Queiroz (2002) recomendaram temperatura de digestão de $350^{\circ} \mathrm{C}$. Nestas mesmas condições, Cecchi (2003) sugeriu temperaturas de digestão variando de 370 a $410^{\circ} \mathrm{C}$. Avaliando-se as diferentes recomendações, faixas de temperatura de 350 a $400^{\circ} \mathrm{C}$ parecem ser adequadas para o processo de digestão em ácido sulfúrico. Neste contexto, a temperatura utilizada pelo laboratório 2 parece ter sido excessiva. Na presença de excesso de sal, o aquecimento excessivo pode causar perda de $\mathrm{N}$ na forma de amônia (CECCHI, 2003).

Considerando-se que no laboratório 2 empregou-se a maior massa de mistura digestora e a maior temperatura, parece se justificar a baixa recuperação de $\mathrm{N}$ oriundo do EDTA (Tabela 5). Ressalta-se que entre os padrões aqui utilizados, o
EDTA apresenta estrutura orgânica complexa, contendo 10 átomos de carbono por molécula, demandando a ocorrência das reações do processo de digestão de forma mais intensa em comparação aos demais padrões, com estrutura mais simples. Assim, a etapa de digestão parece constituir elemento crítico à análise no laboratório 2 .

Variações entre laboratórios também são observadas no processo de destilação (Tabela 11). Com relação à solução de hidróxido de sódio, embora haja variação entre laboratórios, todas as concentrações encontram-se próximas das recomendações observadas na literatura (e.g. $45 \%$ p/v, WIDHAM, 1998; 50\% $\mathrm{p} / \mathrm{v}$, SILVA \& QUEIROZ, 2002). O volume de solução de hidróxido de sódio, o volume de solução de ácido bórico e a concentração de ácido bórico variaram de 25 a $50 \mathrm{~mL}$, de 10 a $20 \mathrm{~mL}$ e de 2 a $4 \%$, respectivamente.

A avaliação dos procedimentos associados direta ou indiretamente à quantificação de $\mathrm{N}$ permitiu evidenciar alguns pontos críticos que podem ter contribuído para a variação interlaboratorial.

$\mathrm{O}$ volume final de destilação mostrou-se variado entre todos os laboratórios e dentro de dois laboratórios (Tabela 11). $\mathrm{O}$ volume final do processo de destilação deve ser suficiente para garantir que toda a amônia produzida reaja com o ácido bórico. Contudo, a variabilidade no volume afeta a tonalidade da solução por causar maior ou menor diluição dos indicadores.

Considerando-se que ponto final de titulação é definido visualmente, variações na tonalidade por diferenças no volume podem ocasionar erros de identificação do ponto final, ampliando, principalmente, a variação intralaboratorial. Desta forma, a padronização do volume de destilação é desejada para a correta condução do processo analítico. 
Tabela 10. Características gerais do processo de digestão em função dos diferentes laboratórios

\begin{tabular}{|c|c|c|c|c|c|c|c|c|c|c|c|}
\hline \multirow{2}{*}{ Laboratório } & \multicolumn{11}{|c|}{ Característica $^{1}$} \\
\hline & MA & $\mathrm{VA}$ & TA & $\overline{\mathrm{RAA}}$ & SAL & $\mathrm{CM}$ & $\mathrm{RSC}$ & MMD & RMDA & RMDAM & $\mathrm{TD}$ \\
\hline 1 & 250 & 5 & $\mathrm{~T}$ & 2 & $\mathrm{Na}$ & $\mathrm{Cu}$ & $10: 1$ & 2,5 & 0,5 & 1,0 & 400 \\
\hline 2 & 500 & 10 & $\mathrm{~T} / \mathrm{PA}$ & 2 & $\mathrm{Na}$ & $\mathrm{Cu}$ & $10: 1$ & 3 & 0,3 & 0,6 & 430 \\
\hline 3 & 100 & 5 & $\mathrm{PA}$ & 5 & $\mathrm{~K}$ & $\mathrm{Cu}$ & $62,5: 1$ & 2,5 & 0,5 & 2,5 & 350 \\
\hline 4 & 300 & 10 & PA & 3,3 & $\mathrm{Na}$ & $\mathrm{Cu}$ & 10:1 & 3 & 0,3 & 1,0 & 350 \\
\hline 5 & 200 & 4 & PA & 4 & $\mathrm{Na}$ & $\mathrm{Cu}+\mathrm{Se}$ & $10,9: 1$ & 1 & 0,3 & 1,0 & 400 \\
\hline 6 & 250 & 5 & PA & 2 & $\mathrm{Na}$ & $\mathrm{Cu}$ & $9: 1$ & 1,5 & 0,3 & 0,6 & 400 \\
\hline 7 & 200 & 5 & PA & 2,5 & $\mathrm{~K}$ & $\mathrm{Cu}$ & $9: 1$ & 2,5 & 0,5 & 1,25 & 400 \\
\hline
\end{tabular}

${ }^{1}$ MA, massa de amostra (mg); VA, volume de ácido sulfúrico (mL); TA, tipo de ácido (T, grau técnico; PA, puro); RAA, relação ácido/amostra (mL/100 mg); SAL, tipo de sal utilizado para elevação da temperatura de ebulição do ácido sulfúrico ( $\mathrm{Na}$, sulfato de sódio; $\mathrm{K}$, sulfato de potássio); $\mathrm{CM}$, catalisador metálico utilizado (Cu, sulfato de cobre; $\mathrm{Cu}+\mathrm{Se}$, mistura de sulfato de cobre e selenito de sódio na proporção de 15:1); RSC, relação entre o sal e catalisador metálico na mistura digestora (g/g); MMD, massa de mistura digestora (g); RMDA, relação mistura digestora/ácido sulfúrico (g/mL); RMDAM, relação mistura digestora/amostra (g/100 mg); TD, temperatura máxima de digestão $\left({ }^{\circ} \mathrm{C}\right)$.

Tabela 11. Características gerais dos processos de destilação e titulação em função dos diferentes laboratórios

\begin{tabular}{|c|c|c|c|c|c|c|c|c|c|c|}
\hline \multirow{2}{*}{ Laboratório } & \multicolumn{10}{|c|}{ Característica $^{1}$} \\
\hline & $\mathrm{CNaOH}$ & $\mathrm{VNaOH}$ & $\mathrm{VAB}$ & $\mathrm{CAB}$ & VFD & IND & FUB & CGB & TFD & FAA \\
\hline 1 & $1: 2$ & 25 & 20 & 4 & 100 & $\mathrm{VM} / \mathrm{VB}$ & PP & BV & $\mathrm{RC}$ & $\mathrm{NP}$ \\
\hline 2 & 40 & $40-50$ & 20 & 4 & $125-150$ & VM & SFD & NI & $\mathrm{RC}$ & NP \\
\hline 3 & 50 & 25 & 10 & 2 & 75 & VM/VB & PPA & BV & NI & NP \\
\hline 4 & 50 & 25 & 10 & 4 & 50 & $\mathrm{VM} / \mathrm{VB}$ & PP & BV & NI & NP \\
\hline 5 & $0,6: 1$ & 20 & 10 & 2 & V & $\mathrm{VM} / \mathrm{VB}$ & NU & $\mathrm{NU}$ & $\mathrm{RE}$ & NU \\
\hline 6 & 50 & 25 & 10 & 4 & 100 & NI & PP & BV & NI & NP \\
\hline 7 & $1: 2$ & 25 & 10 & 4 & 100 & $\mathrm{VM} / \mathrm{VB}$ & $\mathrm{PP}$ & BV & NI & NU \\
\hline
\end{tabular}

$\mathrm{CNaOH}$, concentração da solução de hidróxido de sódio (os valores dados na forma de relação indicam a massa de hidróxido de sódio em relação ao volume de água; os valores numéricos simples indicam \% p/v); $\mathrm{VNaOH}$, volume da solução de hidróxido de sódio por amostra (mL); VAB, volume da solução de ácido bórico por amostra $(\mathrm{mL})$; CAB, concentração de ácido bórico na solução (\% p/v); VFD, volume final de destilação (mL; V, variável em função da amostra); IND, indicador utilizado na solução de ácido bórico (VM, vermelho de metila; VB, verde de bromocresol; NI, não identificável); FUB, frequência de uso de brancos (PP, por partida de digestão; SFD, sem frequência definida; PPA, por partida de ácido clorídrico preparada; NU, não utiliza); CGB, conceito geral de branco (BV, branco verdadeiro produzido com todos os reagentes a partir da etapa de digestão; NU, não utiliza; NI, não identificável); TFD, tonalidade final para titulação (RC, rósea clara; RE, rósea escura; NI, não identificável); FAA, frequência de aferição do ácido clorídrico (NP, na preparação da solução; NU, não utiliza aferição) 
A este comportamento adiciona-se o fato de que em quatro laboratórios não foram evidenciados padrões que permitam identificar o ponto final da titulação, o que certamente afeta a qualidade dos resultados obtidos. Em um laboratório, utiliza-se tonalidade escura como ponto final de titulação. Este procedimento não se amostra adequado, pois o indicador se mostra pouco sensível a variações no volume de ácido quando tonalidade escura é alcançada. Em somente dois laboratórios tonalidade clara foi estabelecida como ponto final de titulação.

A utilização de branco mostra-se essencial na condução de análises de PB, pois a partir deste contabilizam-se as possíveis interferências ambientais, humanas e/ou por influência dos reagentes sobre os resultados. Nos casos em que o mesmo não é utilizado, estas interferências poderão consumir ácido no processo de titulação, o qual será erroneamente contabilizado como $\mathrm{N}$ nos cálculos posteriores, gerando viés.

O conceito de branco deve ser aplicado de forma "verdadeira", isto é, a alíquota em branco deve receber todos os reagentes e passar por todas as etapas analíticas do processo. Em cinco dos laboratórios avaliados este conceito foi aplicado corretamente. Dos demais laboratórios, em um desses não se utiliza branco e em outro o conceito não pôde ser definido a partir das repostas apresentadas. Dos seis laboratórios em que o branco é utilizado, somente quatro utilizam brancos a cada partida de análises (Tabela 11).

O preparo da solução de ácido clorídrico constitui ponto crítico do processo devido à sua importância na quantificação do $\mathrm{N} \mathrm{e}$ em função do comprometimento da exatidão da solução face à volatilidade do ácido clorídrico concentrado. Sua aferição deve ser realizada por intermédio de soluções exatas, como aquelas produzidas a partir do carbonato de sódio (SILVA \&
QUEIROZ, 2002). A não padronização do ácido clorídrico implicará viés sobre os resultados, o qual será diferente a cada nova solução produzida. Em dois dos laboratórios a padronização do ácido clorídrico não é realizada (Tabela 11).

Conclui-se que os teores de PB estimados pelos laboratórios participantes utilizando o método de Kjeldhal são dependentes do efeito de interação do material avaliado e do laboratório, possível reflexo das muitas diferenças entre os procedimentos adotados por cada laboratório.

\section{AGRADECIMENTOS}

Ao INCT-Ciência Animal, ao CNPq e à
$\begin{aligned} & \text { FAPEMIG (PPM) pelo financiamento da } \\ & \text { pesquisa. }\end{aligned}$

\section{REFERÊNCIAS}

BARBIN, D. Componentes de

variância: teoria e aplicações. 2.ed.

Piracicaba: FEALQ, 1993. 120p.

CECCHI, H.M. Fundamentos teóricos e práticos em análise de alimentos. 2.ed.

Campinas: Unicamp, 2003. 207p.

FAITHFULL, N.T. Methods in agricultural chemical analysis. A practical handbook. New York: CABI Publishing, 2002. 266p.

HORWITZ, W.A.; ALBERT, R.; DEUTSCH, M.J.; THOMPSON, J.N. Precision parameters of methods of analysis required for nutrition labeling. Part I. Major nutrients. Journal of AOAC International, v.73, p.661-680, 1990. 
Rev. Bras. Saúde Prod. Anim., Salvador, v.17, n.4, p.696-709 out./dez., 2016 http://www.rbspa.ufba.br

MERTENS, D.R. Challenges in measuring insoluble dietary fiber.

Journal of Animal Science, v.81, p.3233-3249, 2003.

POMERANZ, Y.; MELOAN, C.E. Food analysis: theory and practice. Westport: AVI, 1978. 710p.

SILVA, D.J.; QUEIROZ, A.C. Análise de Alimentos: Métodos químicos e biológicos. 3.ed. Viçosa: UFV, 2002. $235 \mathrm{p}$.

THIEX, N.; MANSON, A.

Determination of crude protein in animal feed, forage, grain, and oilseeds by using block digestion with a copper catalyst and steam distillation into boric acid: collaborative study. Journal of AOAC International, v.85, p.309-317, 2002.

WINDHAM, W.R. Animal feed. In: ASSOCIATION OF OFFICIAL ANALYTICAL CHEMISTS - AOAC (Ed.). Official Methods of Analysis of AOAC International. vol. 1., 16 ed., 4 rev. Gaithersburg, 1998.

Data de recebimento: 17/09/2015

Data de aprovação: 30/08/2016 